\title{
Frequência de lesões sugestivas de toxoplasmose ocular em uma população rural do Estado do Rio de Janeiro
}

\author{
Frequency of lesions suggestive of ocular toxoplasmosis among \\ a rural population in the State of Rio de Janeiro
}

\author{
Ana Luisa Quintella do Couto Aleixo ${ }^{1}$, Eliezer Israel Benchimol ${ }^{1}$, Elisabeth de Souza Neves ${ }^{1}$, \\ Cassius Schnell Palhano Silva ${ }^{1}$, Léa Camillo Coura ${ }^{1}$ e Maria Regina Reis Amendoeira ${ }^{2}$
}

\begin{abstract}
RESUMO
Para determinar a prevalência da toxoplasmose ocular na população em geral do bairro de Santa Rita de Cássia, Barra Mansa, RJ, foi realizado um estudo seccional no qual 1.071 indivíduos foram submetidos a testes sorológicos (IgG e IgM anti-Toxoplasma) e a exame físico e oftalmológico. 0 diagnóstico da toxoplasmose ocular presumida foi baseado em critérios clínicos, sorológicos e aspecto da lesão retinocoroidiana. As lesões foram classificadas em três tipos morfológicos: 1. Limites marcados com halo de hiperpigmentação e área de atrofia coriorretiniana central. 2. Halo hipopigmentado e área central hiperpigmentada e 3. Hiperpigmentadas ou hipopigmentadas. A prevalência de lesões cicatrizadas compatíveis com toxoplasmose ocular foi de 3,8\% na população em geral e 5,8\% entre os indivíduos com sorologia positiva para Toxoplasma gondii (65,9\% dos indivíduos analisados), com predominância de: lesões do tipo 1 (41,5\%), sexo feminino (68,3\%), periféricas $(58,5 \%)$ e menores que 3 diâmetros de disco $(87,8 \%)$.
\end{abstract}

Palavras-chaves: Toxoplasmose ocular. Retinocoroidite. Uveíte. Prevalência.

\begin{abstract}
To determine the prevalence of ocular toxoplasmosis among the general population of the district of Santa Rita de Cassia, Barra Mansa, State of Rio de Janeiro, a cross-sectional study on 1,071 individuals was performed. These subjects underwent serological tests (anti-Toxoplasma $\mathrm{IgG}$ and $\mathrm{IgM}$ ) and physical and ophthalmological examinations. The diagnosis of presumed ocular toxoplasmosis was based on clinical and serological criteria and the appearance of the retinochoroidal lesion. The lesions were classified into three morphological types: 1 . Limits marked with a halo of hyperpigmentation and an area of central chorioretinal atrophy; 2 . Hypopigmented halo and hyperpigmented central area; and 3. Hyperpigmented or hypopigmented. The prevalence of healed lesions compatible with ocular toxoplasmosis was $3.8 \%$ among the general population and 5.8\% among individuals who were seropositive for Toxoplasma gondii (65.9\% of the individuals evaluated). Type-1 lesions (41.5\%), female sex (68.3\%), peripheral lesions (58.5\%) and lesions smaller than three disc diameters predominated.
\end{abstract}

Key-words: Ocular toxoplasmosis. Retinochoroiditis. Uveitis. Prevalence.

A toxoplasmose é uma zoonose de elevada prevalência sendo poucos os estudos em base populacional de suas manifestações oculares. O Toxoplasma gondii é o agente etiológico mais frequente das uveítes posteriores em várias partes do mundo, inclusive no Brasil ${ }^{3611}$. A prevalência de lesões oculares no Brasil é bastante variável de acordo com a área estudada. Em estudantes da Cidade de Natal (RN), a prevalência encontrada foi de 1,2\%, já em Erichim no sul do Brasil foi de 17,7\% 8121318 . Outros trabalhos no Brasil demonstraram prevalências dentro desta faixa de variação dependendo da área geográfica, condições ambientais e faixa etária estudada ${ }^{2}{ }^{4}$. Em Maryland e no Estado do

1. Instituto de Pesquisa Clínica Evandro Chagas, Fundação Oswaldo Cruz, Rio de Janeiro, RJ. 2. Escola Nacional de Saúde Pública, Fundação Oswaldo Cruz, Rio de Janeiro, RJ. Instituto Oswaldo Cruz, Fundação Oswaldo Cruz, Rio de Janeiro, RJ. Endereço para correspondência: Dra. Ana Luisa Quintella do Couto Aleixo. Dept $^{\circ}$ de Oftalmologia/IPEC/FIOCRUZ. Av. Brasil 4365, Manguinhos, 21040-360 Rio de Janeiro, RJ.

e-mail: alquintella@hotmail.com

Recebido para publicação em 01/07/2008

Aceito em 05/03/2009
Alabama (EUA), a prevalência de lesões de retinocoroidite sugestivas de toxoplasmose na população em geral foi de $0,6 \%{ }^{21}$.

Os estudos sobre a prevalência da toxoplasmose ocular na população geral são escassos e nenhum trabalho neste sentido foi encontrado na literatura no que concerne à região sul do Estado do Rio de Janeiro

0 presente trabalho foi proposto para conhecer a prevalência da infecção ocular por Toxoplasma gondii nas suas fases ativas e cicatriciais sob demanda da comunidade do bairro de Santa Rita de Cássia, Município de Barra Mansa, RJ, após o diagnóstico de casos de toxoplasmose ocular.

\section{PACIENTES E MÉTODOS}

Foi realizado um estudo seccional em 1.071 indivíduos de uma população estimada de 1.300 habitantes do bairro de Santa Rita de Cássia, Município de Barra Mansa localizado no sul do Estado do Rio de Janeiro, na região do médio Paraíba, que têm como principal atividade a horticultura. 
Todos os dados foram coletados em duas semanas no mês de novembro de 2004 .

A população incluiu indivíduos de ambos os sexos, de todas as raças, com faixa etária de seis meses a 88 anos de idade sem distinção social ou de estado de saúde.

Foram convidadas a participar do estudo todas as pessoas com mais de 18 anos que assinaram o Termo de Consentimento Livre e Esclarecido, menores de 18 anos somente participaram com concordância e assinatura do termo por seu responsável legal. Foram excluídos os indivíduos com suspeita de ângulo estreito da câmara anterior (pelo risco de fechamento angular e glaucoma agudo com a midríase), indivíduos que não permitiram exame de fundo de olho ou que a retina não pôde ser visualizada por opacidade dos meios. A suspeita de ângulo estreito foi levantada sempre que observada câmara anterior rasa através da iluminação direta focal e corte óptico na lâmpada de fenda.

A população estudada é predominantemente rural, dedicada a horticultura e a região estudada está localizada nas seguintes coordenadas geográficas: $22^{0} 32^{\prime} 25,19^{\prime \prime} \mathrm{S}$ e $44^{0} 10^{\prime} 35,33$ ” numa altitude de $381 \mathrm{~m}$. 0 clima é mesotérmico, com verões quentes e chuvosos e inverno seco. A umidade relativa do ar fica em torno de $77 \%$, a temperatura média mínima é de $16^{\circ} \mathrm{e}$ a média é de $28^{\circ}$.

0 projeto foi aprovado pelo Comitê de Ética em Pesquisa do Instituto de Pesquisa Clínica Evandro Chagas - Fundação Oswaldo Cruz, parecer número 0013.1.011.009-04.

Exames clínicos. Físico e oftalmológico: o exame físico foi realizado por dois médicos infectologistas, na busca de sinais e sintomas sugestivos da toxoplasmose aguda.

0 exame oftalmológico incluiu a medida da acuidade visual pela tabela de Snellen, biomicroscopia de segmento anterior e oftalmoscopia indireta sob midríase. Todos os indivíduos foram examinados por dois oftalmologistas e aqueles que apresentaram alterações fundoscópicas foram revistos por um terceiro.

Para fins do presente trabalho, foram consideradas lesões compatíveis com toxoplasmose: 1. Focos de retinocoroidite em atividade, caracterizados por lesões da coróide e da retina brancacentas com ou sem exsudação vítrea e 2. Focos de retinocoroidite cicatrizados com as seguintes características ${ }^{17}$ : a) Tipo 1. Limites bem marcados com halo de hiperpigmentação e área de atrofia coriorretiniana central (Figura 1); b) Tipo 2. Lesões com halo hipopigmentado e área central hiperpigmentada (Figura 2); c) Tipo 3. Lesões hiperpigmentadas ou hipopigmentadas compatíveis com hiperplasia ou atrofia do epitélio pigmentar retiniano.

As lesões foram chamadas de centrais se estivessem localizadas entre as grandes arcadas vasculares, sendo subdivididas ainda em: maculares, perimaculares e peridiscais. As lesões periféricas tinham sua localização definida nas áreas nasal e temporal, superior e inferior. Na análise das características das lesões oculares, não foi considerada a origem congênita ou adquirida da doença.

Diagnóstico sorológico: amostras de sangue de cada indivíduo foram coletadas na ocasião do exame médico e o soro foi acondicionado em temperatura de $-20^{\circ} \mathrm{C}$ até o momento

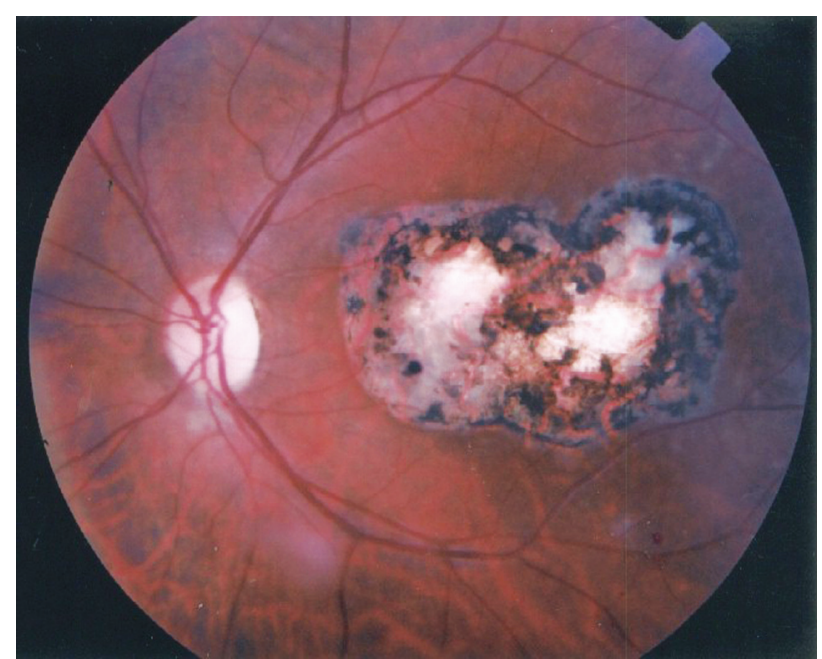

FIGURA 1

Lesão de retinocoroidite tipo 1 (Limites bem marcados com halo de hiperpigmentação e área de atrofia coriorretiniana central).

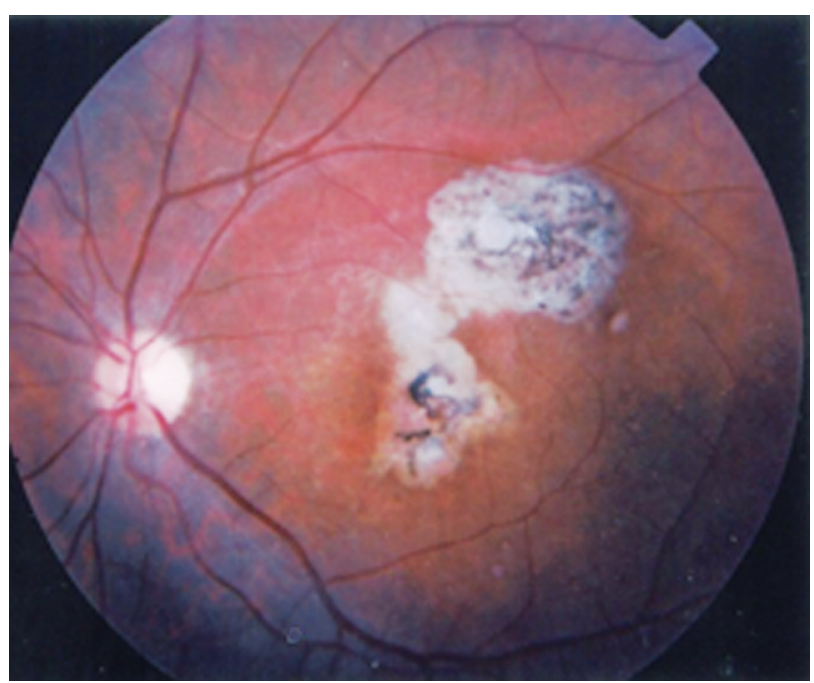

FIGURA 2

Lesão de retinocoroidite tipo 2 (Lesões com halo hipopigmentado e área central hiperpigmentada).

do processamento do teste sorológico. Todos os soros foram submetidos aos seguintes métodos: Teste imunoenzimático (ELISA), segundo Uchôa e cols²3, teste de imunofluorescência indireta (RIFI), modificada conforme Coutinho e col'5.

Os soros foram considerados positivos na RIFI se os títulos fossem iguais ou maiores que 1:16 e/ou igual ou maior do que 0 cut off da placa no ELISA.

Análise estatística. A estatística descritiva e o teste do qui quadrado, para avaliação de diferenças em proporções entre sexo e presença de retinocoroidite, foram realizados utilizandose o software SPSS (Statistical Package for the Social Sciences, Chicago, versão 11.0). Considerou-se um intervalo de confiança de $95 \%$ e o p-valor < 0.05 como estatisticamente significativo. 


\section{RESULTADOS}

Dos 1.300 habitantes do bairro de Santa Rita de Cássia, Barra Mansa, foram examinados $1.071(82,4 \%)$ indivíduos da população total estimada da região. Tendo sido excluídos 13 $(1,2 \%)$ indivíduos por não colaborarem com o exame de fundo de olho, por catarata que inviabilizava a observação da retina ou por possuírem câmara anterior rasa com contra-indicação à midríase, $5(0,5 \%)$ pela impossibilidade da coleta de sangue para sorologia e os 211 (19,7\%) restantes não atenderam ao convite para participar do trabalho. As 241 crianças menores de 10 anos que foram examinadas não permitiram exame detalhado da periferia, porém em todas foi visualizado o pólo posterior.

A soroprevalência para IgG antiToxoplasma gondii foi de 65,9\% (706 indivíduos). Porém, nenhum paciente apresentou IgM positivo ou sinais e sintomas de toxoplasmose ocular em atividade ou exame físico compatível com a infecção aguda sistêmica.

Nos 505 (47,2\%) indivíduos do sexo masculino, a média de idade foi de 27,2 anos, com desvio padrão de 19,5 e mediana de 24 anos, enquanto nos 566 (52,9\%) do sexo feminino, a idade média foi de 28,4 anos, com desvio padrão de 18,7 e mediana de 26 anos. A idade dos indivíduos estudados variou de 6 meses a 86 anos.

A presença de lesões de retinocoroidite foi mais $(24,4 \%)$ frequiente em pacientes nas faixas etárias dos 21 aos 30 anos e maiores de 60 anos. A Tabela 1 mostra distribuição por faixa etária.

A prevalência de lesões retinianas sugestivas de toxoplasmose ocular foi de 3,8\% na população em geral e de 5,8\% entre os soropositivos para IgG antiToxoplasma gondii.

Entre os 41 indivíduos com lesões sugestivas de toxoplasmose, 29 (70,7\%) eram mulheres e 12 (29,3\%) eram homens, sendo a predominância do sexo feminino estatisticamente significativa $(\mathrm{p}<0,05) ; 24$ eram brancos $(4,8 \%$ entre os 495 sororreagentes brancos), 9 negros (10,7\% entre os 84 negros sororreagentes)

\section{TABELA 1}

Distribuição de 706 indivíduos IgG sororeagentes oriundos do bairro de Santa Rita de Cássia, Barra Mansa-RJ, em novembro de 2004, segundo a presença de lesão de toxoplasmose ocular presumida por faixa etária.

\begin{tabular}{|c|c|c|c|c|c|c|}
\hline \multirow{3}{*}{$\begin{array}{l}\text { Faixa etária } \\
\text { (anos) }\end{array}$} & \multicolumn{6}{|c|}{ Indivíduos IgG } \\
\hline & \multicolumn{2}{|c|}{ pacientes com RC } & \multicolumn{2}{|c|}{ sororreagentes sem lesão } & \multicolumn{2}{|c|}{ total } \\
\hline & $\mathrm{n}^{0}$ & $\%$ & $\mathrm{n}^{\underline{0}}$ & $\%$ & $\mathrm{n}^{\underline{0}}$ & $\%$ \\
\hline $0-10$ & 1 & 2,4 & 53 & 7,8 & 54 & 7,6 \\
\hline $11-20$ & 6 & 14,6 & 117 & 17,6 & 123 & 17,4 \\
\hline $21-30$ & 10 & 24,4 & 124 & 18,6 & 134 & 18,9 \\
\hline $31-40$ & 7 & 17,1 & 134 & 20,1 & 141 & 19,9 \\
\hline $41-50$ & 5 & 12,2 & 104 & 15,6 & 109 & 15,4 \\
\hline $51-60$ & 2 & 4,9 & 65 & 9,8 & 67 & 9,5 \\
\hline$>60$ & 10 & 24,4 & 57 & 8,6 & 67 & 9,5 \\
\hline Idade desconhecida & - & - & 11 & 1,6 & 11 & 1,5 \\
\hline Total & 41 & 5,8 & 665 & 94,2 & 706 & 100,0 \\
\hline
\end{tabular}

RC: retinocoroidite, IgG: imunoglobulina $\mathrm{G}$. e 5 pardos (4,6\% entre os 108 pardos soro-reagentes). Três pacientes não declararam sua origem étnica

Foram encontrados 12 (29,3\%) pacientes com lesão somente na região central da retina, 25 (61\%) somente na região periférica e $4(9,8 \%)$ com lesões central e periférica. A maioria $(53,7 \%)$ dos pacientes apresentava cicatrizes de retinocoroidite únicas e em apenas um dos olhos (51,2\% em olho direito, 31,7\% em olho esquerdo) e do tipo 1 (59,5\%). As Tabelas 2 e 3 mostram a distribuição de acordo com o tipo de lesão e localização. Entre os pacientes com lesões centrais, $6(14,6 \%)$ apresentavam baixa de acuidade visual, destes apenas $1(2,4 \%)$ indivíduo teve perda de visão bilateral. Outros 6 indivíduos apresentavam diminuição da visão, porém a lesão retinocoroidiana não se localizava na região central, portanto não se pode afirmar que foi a lesão a causadora da baixa da visão.

\section{TABELA 2}

Distribuição dos 41 pacientes, com cicatrizes de retinocoroidite toxoplásmica presumida, oriundos do bairro de Santa Rita de Cássia, Barra Mansa-RJ, em novembro de 2004, segundo o tipo de lesão.

\begin{tabular}{lcc}
\hline & \multicolumn{2}{c}{ Pacientes } \\
\cline { 2 - 3 } Tipo de Lesão & $\mathrm{n}^{\circ}$ & $\%$ \\
\hline Apenas Tipo 1 & 17 & 41,5 \\
Apenas Tipo 2 & 10 & 24,4 \\
Apenas Tipo 3 & 5 & 12,2 \\
Tipo 1 e 2 & 2 & 4,9 \\
Tipo 1 e 3 & 4 & 9,8 \\
Tipo 2 e 3 & 2 & $4 ., 9$ \\
Tipo 1, 2 e 3 & 1 & 2,4 \\
\hline
\end{tabular}

\section{TABELA 3}

Distribuição de 41 pacientes, com cicatrizes de retinocoroidite toxoplásmica presumida, oriundos do bairro de Santa Rita de Cássia, Barra Mansa-RJ, em novembro de 2004, segundo a localização retiniana e lateralidade da lesão.

\begin{tabular}{lrr}
\hline & \multicolumn{2}{c}{ Pacientes } \\
\cline { 2 - 3 } Localização e número das lesões oculares & $\mathrm{n}^{0}$ & $\%$ \\
\hline Somente central & 12 & 29,3 \\
Somente periférica & 25 & 61,0 \\
Central e periférica & 4 & 9,8 \\
Uma lesão & 22 & 53,7 \\
Duas lesões & 11 & 26,8 \\
Mais de 2 lesões & 8 & 19,5 \\
Bilateral & 7 & 17,0 \\
Apenas olho direito & 21 & 51,2 \\
Apenas olho esquerdo & 13 & 31,7 \\
\hline
\end{tabular}

\section{DISCUSSÃO}

Poucos estudos descrevem a prevalência de toxoplasmose ocular na população em geral. Sabendo-se que a infecção pelo Toxoplasma gondii varia de acordo com a região e a população estudada devido a fatores socioeconômicos e ambientais ${ }^{21422}$, é provável que exista, também, grandes variações na prevalência da toxoplasmose ocular como já foi verificado por outros autores $^{1121821}$. 
No presente trabalho, a prevalência da toxoplasmose ocular presumida pode ser considerada elevada, quando comparada a outros estudos similares em população em geral ${ }^{921}$.

Em outros estudos sobre a prevalência de lesões oculares inativas atribuíveis ao Toxoplasma gondii em soropositivos podese observar prevalências similares ou mais altas do que a que encontramos: Belo Horizonte, 4,7\% ${ }^{17}$, Venda Nova do Imigrante, Espírito Santo, $11,2 \%^{1}$ e 13,9\% em Belo Horizonte ${ }^{10}$.

No estudo de Erechim/RS, em 1.042 indivíduos a prevalência de retinocoroidite toxoplásmica foi de $17,7 \%$ entre aqueles com sorologia positiva para Toxoplasma gondii, sendo considerada a mais alta já descrita ${ }^{8}$.

A predominância de lesões compatíveis com toxoplasmose ocular em mulheres, também foi observada por Melamed ${ }^{13}$. Tal fato, foi corroborado pela análise estatística dos dados, onde a predominância de lesões oculares por Toxoplasma gondii em pacientes do sexo feminino foi significante em relação ao sexo masculino. É importante frisar que os critérios diagnósticos de toxoplasmose ocular utilizados neste estudo foram os mesmos da prática clínica, aspecto da lesão na oftalmoscopia associado à sorologia positiva para Toxoplasma gondii. 0 não isolamento de antígenos ou do próprio protozoário da lesão torna esse diagnóstico presuntivo, já que nenhum exame invasivo foi realizado.

A maior frequiência de lesões de retinocoroidite nas faixas etárias dos 21 aos 30 anos e maiores de 60 anos poderia ser explicada pelo maior número de indivíduos entre 21 e 30 anos e pela maior chance de adquirir a doença nos pacientes mais idosos.

Quanto ao aspectoracial (cordepeledeclaradapelopaciente), observamos uma frequiência maior de lesões retinocoroidianas nos indivíduos da raça negra, com diferença estatisticamente significativa em relação aos brancos e pardos $(\mathrm{p}<0,05)$. Isto suscitaria questionamentos quanto a susceptibilidade genética dos negros ao Toxoplasma gondii e sua patogenicidade ocular e também em relação a outros aspectos epidemiológicos, como nível sócio cultural e econômico deste grupo populacional.

Ao avaliar as características clínicas dos pacientes com cicatriz de retinocoroidite, foi verificado que quanto à localização e lateralidade das lesões no fundo de olho, houve uma predominância (61\%) de pacientes com lesões somente periféricas e unilaterais, sugerindo que esta seja uma característica freqüente no Brasil ${ }^{121720}$.

As lesões tipo 3, embora sejam consideradas inespecíficas, foram incluídas no presente estudo, uma vez que a literatura mostra que lesões atípicas também podem recidivar com quadros típicos ${ }^{19}$. Além disto, foi freqüente a associação destas lesões, tidas como inespecíficas, com lesões tipo 1 (4/41 - 9,8\%), tipo $2(2 / 41-4,9 \%)$ e com os tipos 1 e $2(1 / 41-2,4 \%)$, o que leva a afirmar que este achado deva ser valorizado. Portanto, ao incluir o tipo 3 de lesão diminuiu-se a especificidade e aumentouse a sensibilidade do diagnóstico. Os pacientes não foram testados para sífilis ou tuberculose assim como foi feito em outros estudos ${ }^{8}$. Embora tenhamos realizado exame clínico nos pacientes, às vezes não é possível descartar esses diagnósticos diferenciais.
A prevalência encontrada em nosso estudo foi alta para os padrões detectados em outras partes do mundo ${ }^{21}$; porém está dentro da variação observada no Brasil ${ }^{1710} 19$.

\section{REFERÊNCIAS}

1. Abreu MT, Belfort Jr R, Garcia AR, Muccioli C, Soriano E, Nussenblat R, Silveira C. Toxoplasmose ocular em Venda Nova do Imigrante, ES, Brasil. Arquivos Brasileiros de Oftalmologia 61:540-545,1998.

2. Amendoeira MRR, Sobral CAQ, Teva A, Lima JN, Klein CH. Serological survey of Toxoplasma gondii infection in isolated Amerindians, Mato Grosso. Revista da Sociedade Brasileira de Medicina Tropical 36: 671-676, 2003

3. Benchimol E, Moreira RB. Toxoplasmose Ocular. Anais da Academia Nacional de Medicina 155: 226-228, 1995.

4. Costa MIC. Aspectos Clínicos e epidemiológicos da Toxoplasmose ocular presumida em zona rural do Estado de Minas Gerais. Dissertação de Mestrado. Santa casa de Misericórdia, Belo Horizonte, 2003.

5. Coutinho SG, Andrade CM, Malvar GS, Ferreira LF. Análise comparativa entre a sensibilidades da reação indireta de anticorpos fluorescentes e da reação de Sabin-Feldman na pesquisa de anticorpos séricos para a toxoplasmose. Revista da Sociedade Brasileira de Medicina Tropical 4:315-325, 1970.

6. Fernandes LC, Orefice F. Aspectos clínicos e epidemiológicos das uveítes em serviço de referência em Belo Horizonte 1970-1993. Revista Brasileira de Oftalmologia 55:569-578, 1995.

7. Garcia CAA, Orefice F, Oliveira Lyra C, Gomes AB, Franca M, Garcia Filho CAA. Socioeconomic conditions as determining factors in the prevalence of systemic and ocular toxoplasmosis in Northeastern Brazil. Ophthalmic Epidemiology 11: 301-317, 2004

8. Glasner PD, Silveira C, Kruszon-Moran D, Martins MC, Burnier Júnior M, Silveira S, Camargo ME, Nussenblatt RB, Kaslow RA, Belfort Júnior R. An unusually high prevalence of ocular toxoplasmosis in southern Brazil. American Journal of Ophthalmology 114: 136-144, 1992.

9. Maetz HM, Kleinstein RN, Federico D, Wayne J. Estimated prevalence of ocular toxoplasmosis and toxocariasis in Alabama. Journal of Infectious Diseases 156: 414, 1987.

10. Maestrini AA, Oréfice F. Tese de Doutorado: Aspectos Clínicos e epidemiológicos da população escolar do Município de Rio Acima - Região metropolitana de Belo Horizonte - Minas Gerais. Faculdade de Medicina da Universidade Federal de Minas Gerais, Belo Horizonte, 1995.

11. Martins MC, Silveira C, Jamra LF, Barros PM, Belfort Jr R, Rigueiro MP, Neves RA. Isolamento do Toxoplasma gondii em carnes e derivados provenientes da região endêmica de toxoplasmose ocular de Erechim, Rio Grande do Sul. Arquivos Brasileiros de Oftalmologia 53:60-66, 1990

12. Melamed J. Peculiaridades da Toxoplasmose ocular no Rio Grande do Sul Arquivos Brasileiros de Oftalmologia 51: 197-198, 1988.

13. Melamed J. Retinocoroidite Toxoplásmica. Tese de Doutorado. Universidade Federal de Minas Gerais, Belo Horizonte, 1991.

14. Melamed J. Toxoplasmosis: Soroepidemiological Structure in Southern Brasil. Proceedings of he Fifth International Symposium, Buenos Aires, p. 269-272, 2000 .

15. Melamed J, Guntzel I, Lindenmeyer R. Uveitis in Southern Brasil. In: Proceedings of the Fifth International Symposium, Buenos Aires, p. 247-250, 2000.

16. Melamed J, Souza E, Caramori C, Kauer C. Toxoplasmose manifestações oculares. Revista da Associação Médica do Rio Grande do Sul, 32: 163-169, 1988.

17. Oréfice F. Uveíte Clínica e Cirúrgica texto e atlas. $2^{\text {a }}$ edição. Editora Cultura Médica, p. 703-704, 2005.

18. Silveira C, Belfort Jr R, Burnier Jr M, Nussemblat R. Acquired toxoplasmic infection as the cause of retinocoroidititis in families. American Journal of Ophthalmology. 196:362-364, 1988

19. Silveira C, Belfort Jr R, Muccioli C, Abreu MT, Martins MC, Victora C, Nussenblatt RB, Holland GN. A follow-up study of Toxoplasma gondi infection in southern Brazil. American Journal of Ophthalmology 131: 351-354, 2001. 
20. Silveira CAM. Estudo da toxoplasmose ocular na região de Erechim, RS. Tese de Doutoramento. Escola Paulista de Medicina, Universidade Federal de São Paulo, São Paulo, 1997.

21. Smith RE, Ganley JP. Ophthalmic survey of a Community-Abnormalities of the fundus. American Journal of Ophthalmology 74:1126, 1972.

22. Spalding SM, Amendoeira MRR, Klein CH, Ribeiro LC. Serological screening and toxoplasmosis exposure factors among pregnant women in South of Brazil. Revista da Sociedade Brasileira de Medicina Tropical 38: 173-177, 2005.

23. Uchôa CMA, Duarte R, Laurentino-Silva V, Alexandre GMC, Ferreira HG, Amendoeira MRR. Padronização de ensaio imunoenzimático para pesquisa de anticorpos das classes IgM e IgG anti-Toxoplasma gondii e comparação com a técnica de imunofluorescência indireta. Revista da Sociedade Brasileira de Medicina Tropical 32: 661-669, 1999. 